\title{
Impurity-free disordering of InAs/InP quantum dots
}

\author{
S. Barik, ${ }^{\text {a) }}$ L. Fu, H. H. Tan, and C. Jagadish \\ Department of Electronic Materials Engineering, Research School of Physical Sciences and Engineering, \\ The Australian National University, Canberra, Australian Capital Territory 0200, Australia
}

(Received 27 April 2007; accepted 22 May 2007; published online 15 June 2007)

\begin{abstract}
Impurity-free disordering (IFD) of the InAs quantum dots (QDs) capped with either an InP layer or an InGaAs/InP bilayer is studied. The samples are coated with a $\mathrm{SiO}_{2}$ or $\mathrm{TiO}_{2}$ dielectric layer followed by rapid thermal annealing at $700,750,800$, and $850{ }^{\circ} \mathrm{C}$ for $30 \mathrm{~s}$. A large differential energy shift of $157 \mathrm{meV}$ is induced by $\mathrm{SiO}_{2}$ in the QDs capped with an InGaAs/InP bilayer. Contrary to the reported results on the suppression of intermixing of GaAs based QDs by $\mathrm{TiO}_{2}$, the authors find that intermixing of $\mathrm{InAs} / \mathrm{InP}$ QDs is promoted by $\mathrm{TiO}_{2}$. X-ray photoelectron spectroscopy depth profiles show that both In and $\mathrm{P}$ outdiffuse to a $\mathrm{TiO}_{2}$ layer whereas $\mathrm{Ga}, \mathrm{In}$, and $\mathrm{P}$ outdiffuse to $\mathrm{SiO}_{2}$ layer leading to different degrees of intermixing. The results indicate that a group V interstitial diffusion mechanism might be responsible for IFD of InAs/InP QDs. ( 2007 American Institute of Physics. [DOI: 10.1063/1.2748845]
\end{abstract}

Impurity-free disordering (IFD), commonly known as impurity-free vacancy disordering, is a simple yet promising method for monolithic integration of active/passive components of optoelectronic devices. ${ }^{1,2}$ InAs/InP quantum dots (QDs) are promising active materials for high performance optoelectronic devices operating at long wavelength region (1.3-2 $\mu \mathrm{m})$. However, IFD of InAs/InP QDs has not been studied comprehensively and its mechanism needs to be further clarified. Wang et al. ${ }^{3}$ carried out impurity-free group III intermixing of an InAs/InGaAlAs QDs-in-well structure grown on an InP substrate and reported a differential photoluminescence (PL) wavelength shift of $92 \mathrm{~nm}$ after annealing at $800{ }^{\circ} \mathrm{C}$ for $30 \mathrm{~s}$ between $\mathrm{Si}_{x} \mathrm{~N}_{y}$ and $\mathrm{SiO}_{2}$ capped samples. Chia et ll $^{4}$ performed group V intermixing in InAs/InP QDs capped with a $\mathrm{SiO}_{2}$ or $\mathrm{SiN}_{x}$ layer and reported that the maximum differential energy shift between samples capped with the two dielectric layers after annealing at $850{ }^{\circ} \mathrm{C}$ was $90 \mathrm{meV}$. They attributed the energy shift observed in the samples capped with a $\mathrm{SiO}_{2}$ layer to group $\mathrm{V}$ outdiffusion due to microcrack formation during annealing. They observed very little suppression of the energy shift in the samples capped with a $\operatorname{SiN}_{x}$ layer, but suggested that the use of $\mathrm{a} \mathrm{TiO}_{2}$ layer could suppress the energy shift and thereby would maximize the differential energy shift. We have recently reported on the improved thermal stability of the InAs/InP QDs containing a GaAs interlayer. ${ }^{5}$ In this letter, we present the results of IFD of these QDs and show that a $\mathrm{TiO}_{2}$ layer does not suppress IFD of InAs/InP QDs unlike that of InGaAs/GaAs QDs. ${ }^{6}$ To understand the mechanism of IFD in InP based QD structures a detailed study on the outdiffusion of different elements from a QD structure to a dielectric layer is necessary but has not been carried out so far. We present the x-ray photoelectron spectroscopy (XPS) depth profiles of different group III and V elements and show that selective and different amounts of outdiffusion of In, Ga, and $\mathrm{P}$ atoms to the dielectric layers result in varying degrees of intermixing. Our results suggest that a group V interstitial diffusion mechanism is responsible for IFD of InAs/InP QDs. We also report on the effects of an InGaAs top cap layer on IFD of InAs/InP QDs and show that a large differ-

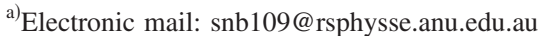

ential energy shift of $157 \mathrm{meV}$ can be obtained by using a top InGaAs cap layer.

InAs QDs are grown on semi-insulating (100) InP substrates using horizontal flow metal organic chemical vapor deposition (MOCVD) at a pressure of 180 mbars. Trimethylindium, trimethylgallium, $\mathrm{PH}_{3}$, and $\mathrm{AsH}_{3}$ are used as precursors, and $\mathrm{H}_{2}$ as the carrier gas. After an oxide desorption step of the substrate at $700{ }^{\circ} \mathrm{C}$ for $10 \mathrm{~min}$ under $\mathrm{PH}_{3}$, the reactor temperature is reduced to $650{ }^{\circ} \mathrm{C}$ and a $700 \mathrm{~nm} \mathrm{InP}$ layer is grown. To minimize the As/P exchange reaction, a $0.6 \mathrm{~nm}$ thick GaAs interlayer is also grown at the same temperature before the growth of a QD layer at $520{ }^{\circ} \mathrm{C}$. The InAs QDs are deposited for $6.5 \mathrm{~s}$ at the rate of $0.16 \mathrm{~nm} / \mathrm{s}$ using a V/III ratio of 8 . The QDs are immediately capped without any growth interruption with a $300 \mathrm{~nm}$ InP layer, while the temperature is increased to $650{ }^{\circ} \mathrm{C}$. In another sample, instead of a $300 \mathrm{~nm} \mathrm{InP}$ layer, an InGaAs/InP bilayer consisting of a $200 \mathrm{~nm} \mathrm{InP}$ layer and a $100 \mathrm{~nm}$ latticematched top InGaAs layer is grown. The QDs have a mean height of $8.2 \mathrm{~nm}$ as measured by atomic force microscopy on uncapped QDs and a mean diameter of $25 \mathrm{~nm}$ as measured by plan-view transmission electron microscopy on capped QDs.

After the MOCVD growth, the samples are deposited with a $180 \mathrm{~nm}$ thick layer of either $\mathrm{SiO}_{2}$ or $\mathrm{TiO}_{2}$ to study IFD of the QDs. $\mathrm{SiO}_{2}$ is deposited using plasma enhanced chemical vapor deposition at room temperature using $\mathrm{N}_{2} \mathrm{O}$ and diluted $\mathrm{SiH}_{4}\left(5 \%\right.$ in $\left.\mathrm{N}_{2}\right) \cdot \mathrm{TiO}_{2}$ is deposited using electron-beam evaporation. After the dielectric layer deposition, the samples are annealed at $700,750,800$, and $850{ }^{\circ} \mathrm{C}$ for $30 \mathrm{~s}$ in argon ambient using a rapid thermal processor. To protect the semiconductor surface from the excessive loss of group $\mathrm{V}$ atoms during annealing, the samples are proximity capped with the fresh substrates having the same group $\mathrm{V}$ atoms.

PL measurements are carried out at $77 \mathrm{~K}$ using the $532 \mathrm{~nm}$ line of a frequency doubled diode pumped solid state laser. The PL signal is collected by a thermoelectrically cooled InGaAs photodetector with a built-in preamplifier after dispersing through a $0.5 \mathrm{~m}$ monochromator.

The outdiffusion of different group III and V atoms from the semiconductor cap layer to the dielectric layer is determined using a VG ESCALAB220i-XL x-ray photoelectron 


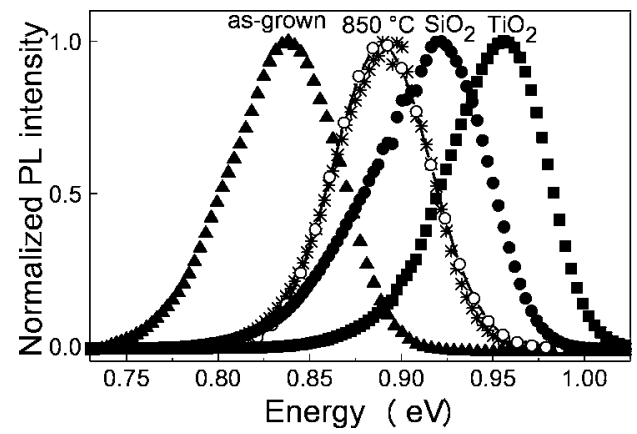

FIG. 1. Normalized $77 \mathrm{~K}$ photoluminescence (PL) spectra of the annealed InAs/InP quantum dots capped with $\mathrm{InP}$ and coated with either $\mathrm{SiO}_{2}$ or $\mathrm{TiO}_{2}$. The PL spectrum of the as-grown sample as well as the PL spectrum of as-grown and annealed sample and its Gaussian fit (the line with open circles) are also shown. The annealing is performed at $850{ }^{\circ} \mathrm{C}$ for $30 \mathrm{~s}$.

spectrometer with a monochromated $\mathrm{Al} K \alpha$ source. For dielectric surfaces, the spectra are calibrated using $\mathrm{C} 1 s$ photoelectron peak corresponding to $\mathrm{C}-\mathrm{C}$ species at the binding energy of $285.0 \mathrm{eV}$. High resolution elemental spectra are acquired for the In $3 d_{5 / 2}, \mathrm{Ga} 2 p_{3 / 2}$, As $2 p_{3 / 2}, \mathrm{P} 2 p_{1 / 2}$, Ti $2 p_{1 / 2}$, Si $2 s$, and $\mathrm{O} 1 s$ photoelectron peaks using an ana lyzer pass energy of $40 \mathrm{eV}$. The area of analysis is about $0.5 \times 0.5 \mathrm{~mm}^{2}$. Depth profiling is carried out by Ar ion sputtering.

The normalized PL spectra of the InP capped samples coated with a $\mathrm{SiO}_{2}$ or $\mathrm{TiO}_{2}$ dielectric layer and annealed at $850{ }^{\circ} \mathrm{C}$ for $30 \mathrm{~s}$ are shown in Fig. 1. The PL spectra of the as-grown sample as well as the as-grown and annealed sample are also shown for reference. All spectra have a dip at $0.895 \mathrm{eV}$ due to atmospheric absorption. After annealing, the PL of the annealed QDs is blueshifted due to thermal interdiffusion compared with that of the as-grown QDs. However, the thermal energy shift is only $54 \mathrm{meV}$. The PL peak energy is further blueshifted for the samples with the $\mathrm{SiO}_{2}$ dielectric layer. Chia et al. ${ }^{4}$ reported that in $\mathrm{SiO}_{2}$ coated InAs/InP QD samples, microcracks formed on the sample surface. A large amount of group $\mathrm{V}$ atoms outdiffused from the sample via the microcracks causing interdiffusion of the QDs. However, we do not observe any microcracks on the sample surface. Another surprising observation is that a $\mathrm{TiO}_{2}$ layer does not suppress the energy shift upon thermal annealing as it does in an InGaAs/GaAs QD system. ${ }^{6}$

Figure 2 shows the energy shift for the samples deposited with a $\mathrm{SiO}_{2}$ or $\mathrm{TiO}_{2}$ layer and annealed at different temperatures for $30 \mathrm{~s}$. The (differential) energy shift for each

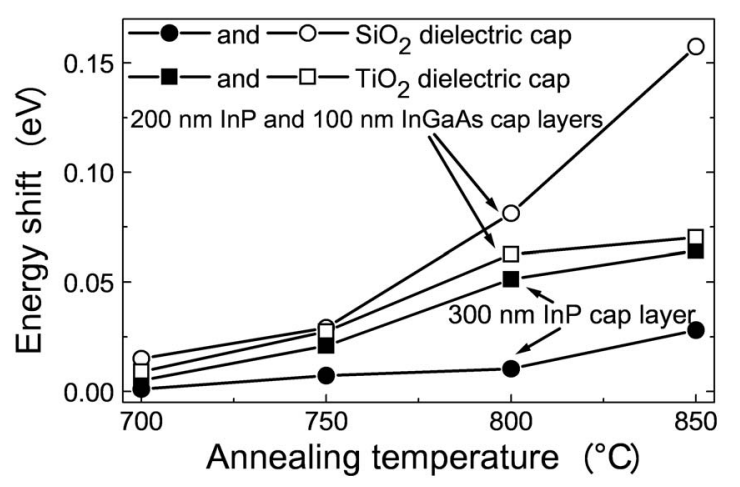

FIG. 2. Energy shifts due to impurity-free disordering of the InAs quantum dots capped with either an InP layer or an InGaAs/InP bilayer and coated with either $\mathrm{SiO}_{2}$ or $\mathrm{TiO}_{2}$. The samples are annealed for $30 \mathrm{~s}$ at different

tration gradient across the QD layer. Teng et al. ${ }^{7}$ proposed
temperatures.
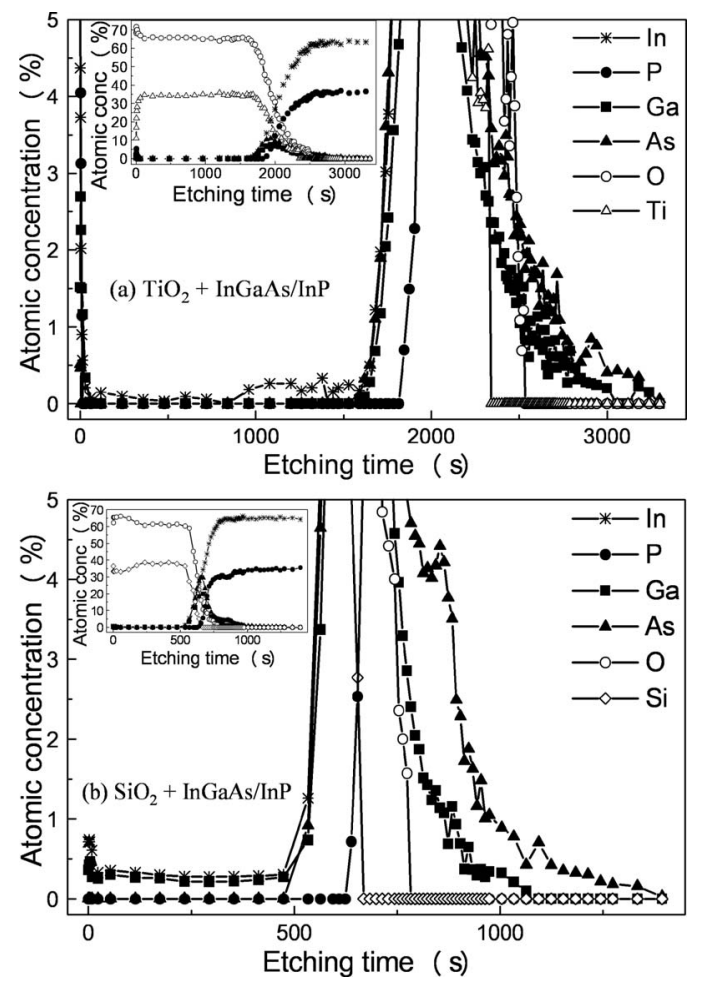

FIG. 3. X-ray photoelectron spectroscopy (XPS) depth profiles of different elements of the InAs quantum dots capped with an InGaAs/InP bilayer and coated with a (a) $\mathrm{TiO}_{2}$ or (b) $\mathrm{SiO}_{2}$ dielectric layer are shown in the insets. The atomic concentration is replotted to show the outdiffusion of atoms to the dielectric layer more clearly. The samples are annealed at $850{ }^{\circ} \mathrm{C}$ for $30 \mathrm{~s}$.

annealing temperature is calculated as the difference between the PL energy peaks of the samples with and without a dielectric layer. As the annealing temperature increases, the energy shift increases. For the QDs capped with the InP layer, a $\mathrm{TiO}_{2}$ layer produces more energy shift than a $\mathrm{SiO}_{2}$ layer. However, for the QDs capped with the InGaAs/InP bilayer, a $\mathrm{SiO}_{2}$ layer gives more energy shift than a $\mathrm{TiO}_{2}$ layer. After annealing at $850{ }^{\circ} \mathrm{C}$, the InGaAs/InP capped QD structure coated with a $\mathrm{SiO}_{2}$ layer shows a large energy shift of $157 \mathrm{meV}$, which is much larger than the reported $\mathrm{SiO}_{2}$ layer and a $\mathrm{SiN}_{x}$ layer coated InAs/InP QD samples having an InP cap layer. ${ }^{4}$

To understand the effects of different cap and coating layers, XPS measurements are carried out. The XPS depth profiles of different elements of InAs QDs capped with the InGaAs/InP bilayer and coated with a $\mathrm{TiO}_{2}$ layer and a $\mathrm{SiO}_{2}$ dielectric layer are shown in the insets of Figs. 3(a) and 3(b), respectively. To show the outdiffusion of different atoms to the dielectric layer more clearly, the atomic concentration is replotted up to $5 \%$ in Fig. 3. The samples are annealed at $850{ }^{\circ} \mathrm{C}$ for $30 \mathrm{~s}$. Figure 3 (a) shows that only In atoms outdiffuse to the $\mathrm{TiO}_{2}$ layer, whereas Fig. 3(b) shows that both In and $\mathrm{Ga}$ outdiffuse to the $\mathrm{SiO}_{2}$ layer. The outdiffusion of these group III atoms to the dielectric layers may lead to the creation of either group III vacancies or group V interstitials in the QD structure. However, the intermixing due to the group III vacancy diffusion will have a minimal role in our QD system. Because of a small thickness of $0.6 \mathrm{~nm}$, the effect of the GaAs interlayer underneath the InAs QD layer is negligible and there is no other group III atomic concentration gradient across the QD layer. Teng et al. ${ }^{7}$ proposed maximum differential energy shift of $90 \mathrm{meV}$ between a 

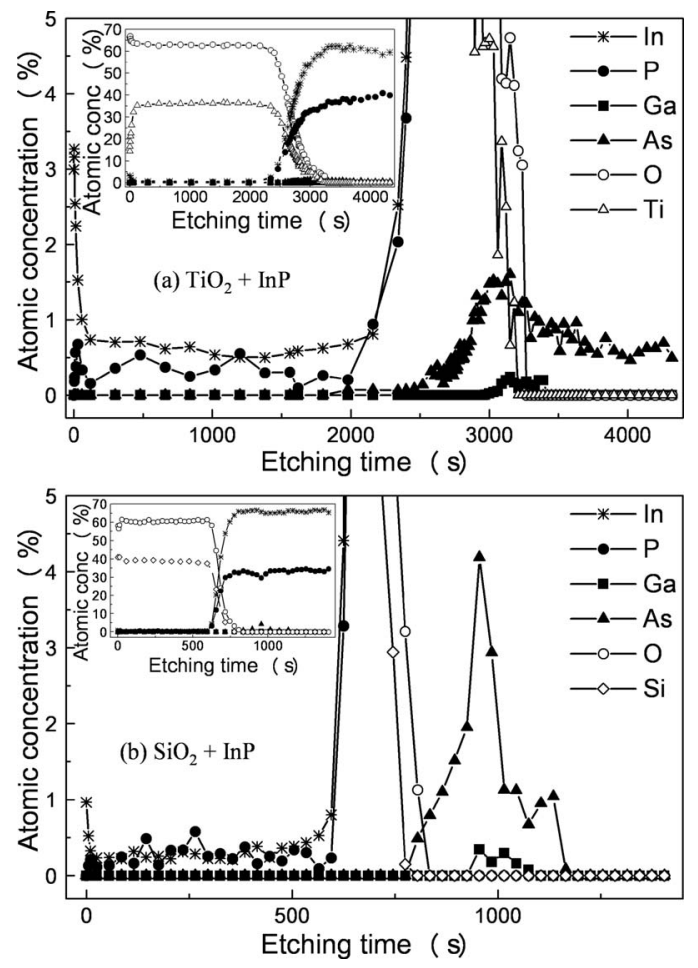

FIG. 4. XPS depth profiles of different elements of the InAs quantum dots capped with an InP layer and coated with a (a) $\mathrm{TiO}_{2}$ or (b) $\mathrm{SiO}_{2}$ dielectric layer are shown in the insets. The atomic concentration is replotted to show the outdiffusion of atoms to the dielectric layer more clearly. The samples are annealed at $850^{\circ} \mathrm{C}$ for $30 \mathrm{~s}$.

that the group $\mathrm{V}$ interstitials generated at the $\mathrm{SiO}_{2}$ and InGaAs surface diffused through the InGaAsP/InP quantum well $(\mathrm{QW})$ heterostructure and led to the enhanced QW intermixing. We also believe that in an InP system, the intermixing by the diffusion of group $\mathrm{V}$ interstitials is a dominant mechanism, as also reported by other groups. ${ }^{8,9}$ The outdiffusion of group III atoms to the dielectric layer results in As interstitials in the InGaAs layer and eventually, P interstitials in the InP layer by the kick-out mechanism. The concentration of P interstitials due to both In and Ga outdiffusion is much higher in the $\mathrm{SiO}_{2}$ coated sample than in the $\mathrm{TiO}_{2}$ coated sample due to only In outdiffusion. As a result, the $\mathrm{SiO}_{2}$ coated sample shows much higher energy shift than the $\mathrm{TiO}_{2}$ coated sample, as shown in Fig. 2. From the insets of Fig. 3, it is also noted that the atomic concentration of In is higher than that of $\mathrm{P}$ in the InGaAs/InP cap layer region. This is due to different sputtering rates of different elements during the depth profiling process. More $\mathrm{P}$ atoms are sputtered preferentially compared to In atoms leading to an In rich surface. However, since all samples are analyzed under the same conditions, we focus our discussion on the effects of elemental outdiffusion from a cap layer to a dielectric layer rather than trying to quantify the exact atomic percentages of different elements.

The XPS depth profiles of different elements of the InAs QDs capped with the InP layer and coated with a $\mathrm{TiO}_{2}$ layer and a $\mathrm{SiO}_{2}$ layer are shown in the insets of Figs. 4(a) and 4(b), respectively. The samples are annealed at $850{ }^{\circ} \mathrm{C}$ for $30 \mathrm{~s}$. The atomic concentration is replotted up to $5 \%$ in Fig. 4. It can be seen that unlike As, P outdiffuses to the dielectric layers. Figure 4(a) shows that more In atoms compared to $\mathrm{P}$ atoms outdiffuse to the $\mathrm{TiO}_{2}$ layer. The outdiffusion of excess In atoms compared to $\mathrm{P}$ atoms results in $\mathrm{P}$ interstitials in the InP cap layer. These P interstitials diffuse through the QD layer causing significant intermixing of the InAs/InP QDs. On the other hand, as shown in Fig. 4(b), the comparable amounts of In and $\mathrm{P}$ outdiffusion to the $\mathrm{SiO}_{2}$ layer result in a small net concentration of $\mathrm{P}$ interstitials leading to a much reduced energy shift, as depicted in Fig. 2.

Our results show that a $\mathrm{TiO}_{2}$ dielectric layer does not suppress interdiffusion as observed in InGaAs/GaAs QD systems, in which intermixing was governed by group III vacancy diffusion. ${ }^{6} \mathrm{TiO}_{2}$ has been recently reported to enhance QW intermixing on $\mathrm{InP} / \mathrm{InGaAs} / \mathrm{InGaAsP}$ heterostructures due to faster In interstitial diffusion. ${ }^{10}$ The thermal expansion coefficient $(\alpha)$ of $\mathrm{TiO}_{2}\left(\alpha=8.2 \times 10^{-6} /{ }^{\circ} \mathrm{C}\right)$ is larger than those of GaAs $\left(\alpha=6.8 \times 10^{-6} /{ }^{\circ} \mathrm{C}\right)$, InGaAs $\left(\alpha=5.69 \times 10^{-6} /{ }^{\circ} \mathrm{C}\right)$, and $\operatorname{InP}\left(\alpha=6.33 \times 10^{-6} /{ }^{\circ} \mathrm{C}\right) . \mathrm{TiO}_{2}$ generates a tensile stress in the semiconductor structure during annealing, and thus inhibits vacancy diffusion but promotes interstitial diffusion. ${ }^{10}$ However, InP and InGaAs become compressive during annealing when it is coated with a $\mathrm{SiO}_{2}$ layer $\left(\alpha=0.52 \times 10^{-6} /{ }^{\circ} \mathrm{C}\right)$ and thereby group $\mathrm{V}$ interstitial diffusion should be inhibited leading to a suppression in energy shift. However, we observe the maximum energy shift in the InGaAs/InP bilayer capped sample with a $\mathrm{SiO}_{2}$ coating. Based on the XPS results, we believe that in our InAs/InP QD system, intermixing is mainly governed by group V interstitial diffusion and influenced by the outdiffusion of different elements to the dielectric layers. The stress may have only a small or insignificant role.

In conclusion, we have studied IFD of the InAs/InP QDs containing a thin GaAs interlayer. Our results show that both $\mathrm{SiO}_{2}$ and $\mathrm{TiO}_{2}$ layers promote IFD of InAs/InP QDs. The XPS and PL studies indicate that group V interstitial diffusion might be the dominant mechanism. XPS depth profiles show that both In and $\mathrm{P}$ outdiffuse to a $\mathrm{TiO}_{2}$ layer whereas $\mathrm{Ga}$, In, and $\mathrm{P}$ outdiffuse to a $\mathrm{SiO}_{2}$ layer. A large differential energy shift of $157 \mathrm{meV}$ is obtained in the QDs capped with an InGaAs/InP bilayer and coated with a $\mathrm{SiO}_{2}$ layer indicating that the use of an InGaAs cap layer in an InAs/InP QD system could be beneficial for the monolithic integration of QD based optoelectronic devices.

The authors are thankful to Bill Gong and Robert Lamb from the University of New South Wales, Sydney, Australia for XPS measurements and fruitful discussions. The financial support from the Australian Research Council is also gratefully acknowledged.

${ }^{1}$ D. G. Deppe, L. J. Guido, J. N. Holonyak, K. C. Hsieh, R. D. Burnham, R. L. Thornton, and T. L. Paoli, Appl. Phys. Lett. 49, 510 (1986).

${ }^{2}$ A. Pepin, C. Vieu, M. Schneider, H. Launois, and Y. Nissim, J. Vac. Sci. Technol. B 15, 142 (1997).

${ }^{3}$ Y. Wang, H. S. Djie, and B. S. Ooi, Appl. Phys. Lett. 88, 111110 (2006). ${ }^{4}$ C. K. Chia, S. J. Chua, S. Tripathy, and J. R. Dong, Appl. Phys. Lett. 86, 051905 (2005).

${ }^{5}$ S. Barik, H. H. Tan, and C. Jagadish, Appl. Phys. Lett. 90, 093106 (2007). ${ }^{6}$ L. Fu, P. Lever, H. H. Tan, C. Jagadish, P. Reece, and M. Gal, Appl. Phys. Lett. 82, 2613 (2003).

${ }^{7}$ J. H. Teng, J. R. Dong, S. J. Chua, M. Y. Lai, B. C. Foo, D. A. Thompson, B. J. Robinson, A. S. W. Lee, J. Hazell, and I. Sproule, J. Appl. Phys. 92, 4330 (2002).

${ }^{8}$ C. Dion, P. J. Poole, S. Raymond, P. Desjardins, and F. Schiettekatte, Appl. Phys. Lett. 89, 131905 (2006).

${ }^{9}$ A. S. W. Lee, M. MacKenzie, D. A. Thompson, J. Bursik, B. J. Robinson, and G. C. Weatherly, Appl. Phys. Lett. 78, 3199 (2001).

${ }^{10}$ A. Francois, V. Aimez, J. Beauvais, M. Gendry, and P. Regreny, Appl. Phys. Lett. 89, 164107 (2006). 